\title{
Alignment of Borderline Personality Disorder and Complex Post-traumatic Stress Disorder With Complex Developmental Symptomatology
}

\author{
Jessica Lawless ${ }^{1} \cdot$ Michael Tarren-Sweeney $^{1}$ (D)
}

Accepted: 18 February 2022

(c) The Author(s) 2022

\begin{abstract}
Cluster analysis of maltreatment-related mental health symptoms manifested by adolescents in foster care suggest the absence of an underlying taxonomic structure. To test this further, we investigated alignment between mental health symptom profiles derived through cluster analysis and nominal diagnosis of Borderline Personality Disorder (BPD) and Complex Post-traumatic Stress Disorder (C-PTSD), among a sample of 230 adolescents in long-term foster care. Nominal DSM-V BPD and ICD-11 C-PTSD caseness was estimated from Child Behaviour Checklist and Assessment Checklist for Adolescents score algorithms, and alignment of case assignment with previously-derived symptom profiles was examined. Nineteen BPD and three C-PTSD nominal cases were identified. Low C-PTSD prevalence reflected low concordance between PTSD and 'disturbances in self organization' (DSO) case assignment. The BPD and C-PTSD cases were aligned to more complex and severe symptom profiles. While the complex and severe presentations identified in the present study included core symptoms and clinical signs of BPD, they were also characterised by clinical-level inattention/over-activity and conduct problems. The present findings provide some support for the validity of the BPD construct for describing complex and severe psychopathology manifested by adolescents in foster care, and no support for the C-PTSD construct. However, the symptom profiles point to high variability in combinations of multiple symptom types that does not conform to traditional definitions of a 'diagnosable' mental disorder. Further research is needed to determine if complex post-maltreatment symptomatology can be validly conceptualised as one or more complex disorders.
\end{abstract}

Keywords Out-of-home care · Developmental psychopathology $\cdot$ Complex disorders $\cdot$ Borderline personality disorder · Complex post-traumatic stress disorder · Classification of mental disorders · Child behavior checklist · Assessment checklist for adolescents

\section{Introduction}

Adolescents residing in long-term out-of-home care (OOHC) (foster, kinship and residential care) have generally experienced substantial, ongoing maltreatment from an early age, culminating in their entry into OOHC. Maltreated children and adolescents, including those placed into statutory care, frequently manifest complex mental health difficulties (DeJong, 2010; van der Kolk

Michael Tarren-Sweeney

michael.tarren-sweeney@ canterbury.ac.nz

1 School of Health Sciences, Canterbury University, Christchurch, New Zealand et al., 2005). These include combinations of: dysregulated affect and behaviour; inattention / over-activity; traumarelated anxiety, hypervigilance and re-experiencing trauma; dissociation and sensory disturbances; negative and unstable perceptions of oneself and others; attachment disorders and other attachment-related interpersonal difficulties; conduct problems, oppositional-defiance, low empathy and aggression; sexual behaviour problems; food maintenance behaviours (hoarding, storing, gorging); restricted, odd and stereotypic behaviours; depression; self-injury; and suicidal ideation and behaviour (TarrenSweeney, 2013b). Cumulative trauma exposure accounts for increasing symptom complexity in both childhood and adulthood (Cloitre et al., 2009). Clinicians therefore struggle to formulate such complex developmental 
symptomatology among these populations (Kocovska et al., 2012; Minnis, 2013). The accuracy and utility of clinical assessments of maltreated children and adolescents are thus compromised by clinicians' inability to formulate complexity, such as resorting to co-morbid diagnosis (D'Andrea et al., 2012; Minnis, 2013); with incorrect formulation compromising the delivery of safe and effective treatments (Spinazzola et al., 2005; van Der Kolk, 2016). This also applies to assessment of complex symptomatology among adults who experienced chronic child maltreatment. Their complex presentations are typically formulated as co-morbid diagnosis of two or more of: Borderline Personality Disorder (BPD) (and other cluster B personality disorders); Post-traumatic Stress Disorder (PTSD); Attention-deficit / Hyperactivity Disorder (ADHD); Bipolar Disorder; Major Depressive Disorder; various dissociative disorders; and substance use disorders (Brand \& Lanius, 2014; Creamer et al., 2001; Frías et al., 2016; Pagura et al., 2010; Weiner et al., 2019).

\section{Diagnostic Constructs that Capture Complex Symptomatology}

\section{Various Iterations of Complex PTSD}

To what extent might complex post-maltreatment psychopathology be conceptualised within a typology of complex disorders? The most important work to date has focussed on differentiating the trauma symptomatology of adults who experienced childhood maltreatment, from that of adults exposed to discrete life threatening events (such as war or natural disasters or single instances of inter-personal violence). The PTSD construct arose from clinical studies of so-called 'war neuroses', and was formalised in the US following the Vietnam war (van der Kolk et al., 2005). The vast majority of cases who develop PTSD following exposure to war or discrete traumatic events in adulthood enjoy normative premorbid psychological development. Furthermore, research has shown that the trauma symptomatology of children, adolescents and adults exposed to severe and prolonged interpersonal trauma extends well beyond PTSD - reflecting more complex, 'developmental' adaptions to extreme stress, including neurobiological capacity to integrate cognitive, emotional and sensory information (van der Kolk, 2005). Indeed, it has been reported that a majority of traumatized children do not meet diagnostic criteria for PTSD (Cook et al., 2005).

To address this, a DSM-IV PTSD field trial was carried out in the early 1990s with the goal of better identifying disorders of extreme stress (Herman, 1992). Following a research review, the field trial workgroup proposed seven symptom categories not encompassed within the PTSD diagnosis, that are observed among people exposed to childhood trauma, women victims of domestic violence, and concentration camp survivors. These were: (a) dysregulation of affect and impulses; (b) alterations in attention or consciousness; (c) alterations in self-perception; (d) alterations in perception of the perpetrator; (e) alterations in relations with others; (f) alterations in systems of meaning; and (g) somatization (van der Kolk et al., 2005). The workgroup proposed a new diagnosis for inclusion in DSM-IV - Disorders of Extreme Stress Not Otherwise Specified (DESNOS) - supported by evidence from the field trials, and informally referred to as Complex PTSD (C-PTSD) (Herman, 1992). While the proposal was not adopted, DSM-IV listed the DESNOS symptoms as associated and descriptive features (American Psychiatric Association, 1994). Complex / developmental trauma constructs were further refined in the 2000s prior to DSM-V's publication, and more recently in the run-up to ICD-11. Cook et al. (2005) refined the DESNOS categories and conceptualised seven domains of complex trauma (attachment; biology; affect regulation; dissociation; behavioural control; cognition; and self-concept). Van der Kolk and other members of the Complex Trauma taskforce of the U.S. National Child Traumatic Stress Network proposed a Developmental Trauma Disorder (DTD) for DSM-V, with four diagnostic criteria: (a) exposure to 'developmental trauma'; (b) triggered pattern of repeated dysregulation in response to trauma cues (affective, somatic, behavioural, cognitive, relational, self-attribution); (c) persistently altered attributions and expectancies (with various specific examples); and (d) functional impairment (educational, familial, peer, legal, vocational) (van der Kolk, 2005).

While DESNOS and DTD were rejected for DSM-IV and DSM-V respectively, a further iteration of the construct, Complex PTSD (C-PTSD), was added to the ICD-11 (World Health Organization, 2020). ICD-11 C-PTSD has somewhat narrower diagnostic criteria than DESNOS and DTD. It is comprised of six symptom clusters grouped into two halves, namely ICD-11 PTSD diagnostic criteria (re-experiencing traumatic event; active avoidance of internal thoughts or external reminders; persistent perceptions of heightened current threat), and three 'disturbances in self organization' (DSO) criteria. The DSO criteria are severe and persistent: (1) problems in affect regulation; (2) beliefs about oneself as diminished, defeated or worthless, accompanied by feelings of shame, guilt or failure related to the traumatic event; and (3) difficulties in sustaining relationships and in feeling close to others (World Health Organization, 2020). C-PTSD diagnosis requires meeting all six PTSD and DSO criteria.

It is notable that the DESNOS relational difficulties category ('alterations in relations with others') does not specify some characteristic relational difficulties observed among maltreated children and adolescents, including secure base distortions such as role reversal and hyper-compliance, 
and various behaviours indicating non-attachment (Boris \& Zeanah, 1999). Similarly, the ICD-11 C-PTSD criterion 'persistent difficulties in sustaining relationships and in feeling close to others' is rather narrowly defined, in that it excludes a range of characteristic relational difficulties seen among this population.

These omissions highlight the challenge in defining the amorphous developmental effects of childhood maltreatment. Chronically maltreated children are often exposed to multiple types of harmful developmental experiences that include physical and/or sexual abuse, as well as emotional abuse and emotional and physical neglect (Tarren-Sweeney, 2016). This goes some way to explaining the complexity of their psychopathology - the determinants of which involve complex interactions between trauma, attachment, genetic and social learning mechanisms.

\section{BPD and Multiple Complex Developmental Disorder}

BPD is an adult personality disorder characterised by a pervasive pattern of instability in affect regulation, impulse control, interpersonal relationships, and self-image (American Psychiatric Association, 2013). Clinical signs include emotional dysregulation, impulsive aggression and impulsivity in other areas, intensely unstable relationships, distorted cognition of relationships, mentalizing difficulties, self-injury, chronic feelings of emptiness or boredom, and chronic suicidal ideation and behaviour (Fonagy \& Bateman, 2006; Newman \& Stevenson, 2005; Trull et al., 2003).

Many of these behaviours and symptoms parallel those manifested by maltreated children and adolescents with complex developmental symptomatology. While BPD is not explicitly conceptualized as an attachment and/or trauma disorder, case control studies and surveys have established a strong link between BPD and childhood maltreatment and other adverse childhood experiences among both adult clinical (Helgeland \& Torgersen, 2004) and adolescent samples (Belsky et al., 2012). One study estimated that $70-80 \%$ of adults with BPD have experienced trauma that is tied to their symptoms (Schmid et al., 2013). In particular, child sexual abuse is a common antecedent of BPD (Infurna et al., 2016; Venta et al., 2012). However, we still lack robust evidence of the developmental trajectory of borderline symptomatology from early childhood trauma through to adult BPD. For example, the long-term stability of emotional and behavioural dysregulation from early childhood through to early adulthood has not been adequately researched. There is also conflicting evidence of the stability of BPD symptoms in adolescence (Lewinsohn et al., 1997), with some studies indicating that adolescents' symptoms are less continuous than those experienced by adults (Chanen et al., 2004; Levy et al., 1999). This may be partly accounted for by adolescents' greater reactivity to present stressors and to the developmental demands of adolescence. This points therefore, to a risk of misidentifying adolescent crises as BPD, particularly among vulnerable youth with maltreatment histories and other co-existing mental health difficulties.

It is notable that several categories / domains specified in DESNOS, DTD and C-PTSD correspond to diagnostic features of BPD. The DESNOS workgroup did not set out to examine the PTSD or DESNOS constructs in relation to BPD (or other Axis I and Axis II disorders) (van der Kolk et al., 2005). However, ICD-11 C-PTSD was explicitly defined to be differentiated from BPD e.g. with respect to 'fear of abandonment' (not a feature of C-PTSD) and maladaptive self-image $(\mathrm{BPD}=$ shifting, unstable; $\mathrm{C}-\mathrm{PTSD}=$ consistently negative) (Brewin et al., 2017; Cloitre et al., 2014). This may partially account for C-PTSD's narrow symptom focus.

The 'borderline' construct has a somewhat messy and controversial history. It originated in psychoanalysis and has referred to various ideas, including psychopathology at the border between neurosis and psychosis, and early-onset childhood psychosis (Ad-Dab'bagh \& Greenfield, 2001). Over time, 'borderline' and BPD labels have acquired a pejorative meaning, describing a class of emotionally dysregulated clients whose symptoms were thought to be untreatable, and who are difficult to engage with (McNab, McCutcheon, \& Chanen, 2021). A BPD diagnosis conveys considerable stigma among mental health clinicians (Sansone \& Sansone, 2013). While we might hope that the development of effective BPD treatments (such as Dialectical Behaviour Therapy and Mentalization-based Treatment) will improve clinicians' attitudes towards people with BPD, applying a BPD label to maltreated adolescents, including those in OOHC, risks further stigmatising an already marginalised population (McNab et al., 2021). Consequently, if 'adolescent BPD' is ever confirmed to be a valid construct, then it warrants a better name - one that more accurately describes its developmental underpinnings. Several decades ago, scholars proposed a reconceptualization of child and adolescent 'borderline' symptomatology as a pervasive developmental disorder, which they termed 'multiplex developmental disorder' and 'multiple complex developmental disorder' (Ad-Dab'bagh \& Greenfield, 2001; Cohen et al., 1986; Towbin et al., 1993). However, this idea has been subjected to very little empirical research.

\section{Empirical Investigation of Complex Developmental Symptomatology among Children and Adolescents}

The above-mentioned diagnostic constructs consist of an historical adult diagnosis (BPD), and attempts to rectify the limitations of the PTSD diagnosis (DESNOS, DTD, C-PTSD). However, traditional conceptualizations of adult trauma syndromes may not be valid for children and adolescents. An alternative approach is to base our conceptualisation of 
complex post-maltreatment symptomatology on empirical data obtained using inductive research methods - rather than deductive modification of existing constructs. There have been remarkably few inductive investigations of complex developmental symptomatology among these populations. The validity of ICD-11 C-PTSD among children and adolescents has been investigated using finite mixture models (Latent Class Analysis; Latent Profile Analysis) that estimate probabilities of case membership of latent classes, notably PTSD and DSO (Ford, 2020; Haselgruber et al., 2020; Perkonigg et al., 2016; Sachser et al., 2017). These studies also had relatively narrow symptom coverage, limited to the PTSD and DSO constructs. They therefore employed a 'top down' confirmatory (i.e. deductive) approach. By contrast, 'bottom up' exploratory (i.e. inductive) analysis of complex symptomatology requires more comprehensive symptom coverage as well as exploratory statistical modelling. Cluster analysis is one such exploratory method, in which case clusters are identified using distance algorithms that seek to identify similar score profiles, without reference to predefined variables (Henry et al., 2005; Steele \& Aylward, 2007). Cluster analyses of carer-report scores across broad symptom groupings (including general externalizing and internalizing symptoms, as well as symptoms more specific to maltreatment) have been carried out on Australian population samples of pre-adolescent children $(N=347)$ (TarrenSweeney, 2013b) and adolescents $(N=230)$ (Tarren-Sweeney, 2021) in family-based (foster and kinship) OOHC. Both child and adolescent cluster analyses yielded mental health profiles differentiated more by symptom severity and complexity, than by symptom specificity - suggesting that complex post-maltreatment symptomatology does not conform to a taxonomy of discrete disorders. Furthermore, $20 \%$ of the child sample (Tarren-Sweeney, 2013b) and 15\% of the adolescent sample (Tarren-Sweeney, 2021) displayed complex maltreatmentrelated symptomatology that is not adequately conceptualized within DSM-5 or ICD-11 classifications.

\section{Alignment of Empirically Derived Symptom Profiles with BPD and C-PTSD}

Whereas there are ongoing efforts to define and classify complex developmental symptomatology using conventional diagnostic classifications, empirical analyses of complex suggest it is not amenable to traditional taxonomic classification. This raises a question about the extent to which existing diagnostic constructs that encompass complex symptoms - notably C-PTSD and BPD - align with empirically derived symptom profiles. The present article reports secondary analyses of the aforementioned Australian adolescent mental health data published in this special issue (Tarren-Sweeney, 2021), designed to address the research question:
"To what extent do DSM-V Borderline Personality Disorder and ICD-11 Complex Post-traumatic Stress Disorder align with complex symptomology manifested by adolescents in foster care?"

\section{Method}

\section{Study Design}

The Children in Care study (CICS) was a prospective epidemiological study of the mental health and developmental risk exposure of children and young people in long-term foster and kinship care, in New South Wales (NSW), Australia. The CICS included a baseline cross-sectional survey of 4-11 year-olds conducted between November 1999 and January $2003(N=347)$; a follow-up survey of those participants conducted in 2009 (by which time they were adolescents); and a cross-sectional survey of additional adolescents carried out in 2011. The second and third of these surveys employed the same study design with a view to yielding a combined $(N=230)$ cross-sectional dataset obtained for a representative sample of adolescents residing in family-based OOHC (foster and kinship) in NSW. Data were collected from mail-out caregiver questionnaires, and from the state child welfare and OOHC database, except that child welfare records were not retrieved for newly-recruited adolescents. The caregiver questionnaire measured participants' mental health, development, education and present status (e.g. type and makeup of present placement, recent life events).

The statutory agency that held legal guardianship of the young people approved the study, while participation in the adolescent survey also required informed consent of both the young person and their carer. Aside from providing informed consent, young people were not directly involved in the study.

The present analyses set out to investigate alignment between nominal BPD and C-PTSD case assignments and the empirically derived symptom profiles, with case assignment being estimated from mental health checklist score algorithms that correspond to diagnostic criteria.

\section{Mental Health Measures}

Adolescent mental health was measured by two carer-report checklists, the Child Behaviour Checklist (CBCL, 2001 profile) (Achenbach \& Rescorla, 2001) and the Assessment Checklist for Adolescents (ACA) (Tarren-Sweeney, 2013a). The CBCL measures common child mental health difficulties across eight empirically derived clinical subscales; two higher-order, broadband scales approximating spectrums of depressive/anxious symptoms (internalizing) and 
disruptive behavioural symptoms (externalizing); and a total problems score that provides a measure of global psychopathology. Additionally, the CBCL has six DSM-oriented scales derived through expert ratings of items' conforming to DSM-IV-TR diagnostic criteria (Achenbach \& Rescorla, 2001). CBCL scale score distributions are delineated into normal, borderline clinical and clinical ranges.

The ACA is a 105-item carer-report mental health rating scale, measuring behaviours, emotional states, traits, and manners of relating to others, as manifested by adolescents residing in OOHC (Tarren-Sweeney, 2013a). It was developed to measure a range of attachment- and trauma-related problems not adequately covered by standard survey instruments, including the CBCL, with most items being derived from the pre-adolescent Assessment Checklist for Children (Tarren-Sweeney, 2007). In addition to a total clinical score, the ACA has seven clinical scales that measure various attachment- and trauma-related symptomatology, derived through factor analysis of 73 core clinical items (a robust 7 -factor model accounted for $51 \%$ of score variance). Four of the factors replicate ACC clinical scales (non-reciprocal interpersonal behaviour; sexual behaviour problems; food maintenance behaviour; and suicide discourse), and three are unique to the ACA (social instability / behavioural dysregulation; emotional dysregulation / distorted social cognition; and dissociation / trauma symptoms). The ACA also contains two low self-esteem scales (low confidence; negative self-image) that were constructed separately to the clinical scales. For each ACA scale, two cut-points were selected to identify young people with 'possible' and 'probable' clinical-level problems. Scores above the higher cutpoints constitute clinical ranges that are highly predictive of psychiatric impairment (highly specific), while scores above the lower cut-point ranging up to and including the high cutpoint constitute elevated ranges that retain high sensitivity for detecting psychiatric impairment. Initial data indicate that the ACA has good content, construct and criterionrelated validity, as well as high internal reliability (TarrenSweeney, 2013a).

\section{Sample Recruitment}

\section{Follow-up Participants}

Of 347 baseline survey participants, 231 remained in courtordered foster or kinship care at follow-up. Of these, 66 were residing in placements that did not have a verifiable contact address, and whose carers could not be located by telephone. There were thus 165 young people that were eligible for inclusion in the follow-up survey, and could be reliably located. Of these, questionnaires were returned for 85 young people, representing a $51.5 \%$ response rate. However, these participants represented only $37 \%$ of baseline participants who remained in care.

\section{Additional Adolescent Survey Participants}

The sampling frame for recruiting additional adolescent survey participants was: young people aged 12 to 17 years residing in non-temporary court-ordered foster and kinship care in New South Wales, Australia; case supervision was provided by the statutory child welfare agency (i.e. not supervised by private agencies); were not part of the baseline survey sample; and whose placement address could be verified. Survey questionnaires were sent to the caregivers of 290 eligible participants residing at verified residential addresses with telephone contact, of which 145 were completed and returned (50\% response rate). Additional adolescent participants did not differ to eligible non-participants in terms of age or gender distributions, geographical location, time in care, or age at entry into care.

\section{Sample Characteristics}

The adolescent survey sample $(N=230)$ consisted of 85 follow-up participants, and 145 newly recruited participants. It included slightly more boys $(54 \%, \mathrm{~N}=125)$ than girls $(46 \%, \mathrm{~N}=105)$. Participants had a mean age of 15.3 years, ranging from 10.7 to 18.6 years. Ethnicity was not reliably recorded in the state database at the time data were collected, and hence it is not reported. Numbers of young people residing in foster and kinship care were $196(85 \%)$ and 34 $(15 \%)$ respectively. While the maltreatment histories of the newly-recruited participants were not retrieved, 79\% and $75 \%$ of the follow-up participants had confirmed prior exposure to abuse (sexual, physical and/or emotional) and neglect respectively, while 55\% had experienced both abuse and neglect. Aggregate sample mean CBCL and ACA scores have been reported previously (Tarren-Sweeney, 2018). Fifty-two percent of girls and $46 \%$ of boys had at least one CBCL syndrome or broadband scale score in the clinical range. Equivalent proportions of young people with any score in the borderline plus clinical ranges were 63\% (girls) and 59\% (boys).

\section{Symptom Profiles}

The companion article (Tarren-Sweeney, 2021) reports two sets of symptom profiles identified among the present sample through cluster analysis. The first cluster analysis identified symptom profiles across eight ACA scales for 113 ACA cases, defined by a clinical range score on one or more of the ACA scales, including the ACA total clinical score. Eight ACA symptom profiles (depicting variation in profile shape and mean scale score elevation) are listed 
in the companion article, while the three most severe and complex profiles (\#6, \#7, and \#8) are listed in Fig. 1. The profiles place cluster mean scores for each of the ACA scales within the four ranges used on the ACA score profile sheets: sub-clinical scores are delineated between normative range and elevated range scores; while clinical-level scores are delineated between clinically indicated range (less severe) and marked clinical range (more severe) scores.

The second cluster analysis sought to identify characteristic symptomatology across a broader range of symptoms, as measured across ten CBCL DSM-oriented and ACA scales, namely: five CBCL DSM-oriented scales (Affective problems, Anxiety problems, Attention-deficit / hyperactivity problems, Oppositional defiant problems, and Conduct problems); and five ACA scales (Nonreciprocal interpersonal behaviour; social instability / behavioural dysregulation; emotional dysregulation / distorted social cognition; dissociation / trauma symptoms; and negative self-image). Cases $(N=141)$ were defined as participants with a clinical range score on any CBCL broadband, CBCL DSM-oriented, or ACA scale. Symptom profiles for 11 CBCL-DSM/ACA clusters (depicting variation in profile shape and overall elevation of scores) are listed in the companion article, while the three most severe and complex profiles (\#9, \#10,\#11) are listed in Fig. 2. For the CBCL-DSM scales, the four symptom severity ranges were defined by CBCL T-score ranges, namely normative (T-scores $<63$, representing scores that are clearly normative); elevated $(\mathrm{T}$-scores $=63-69$, representing sub-clinical scores that are less clearly normative, with the upper end encompassing the CBCL borderline clinical range); clinically indicated (T-scores $=70-73$, the moderate end of the clinical range); and marked clinical ( $\mathrm{T}$-scores $>73$, the severe end of the clinical range).

\section{Defining Nominal BPD and C-PTSD Cases}

Nominal BPD and C-PTSD cases were identified from a multi-step method. The first step was to identify and review all CBCL and ACA items that potentially match each diagnostic criterion. Where the two checklists have an equivalent item, the ACA item was selected. Most of the rejected items lacked sufficient specificity to the diagnostic criterion. The rejected items are listed in a 'supplementary materials' table, along with the reason for rejection. We could not identify any suitable items to measure C-PTSD criterion \#2 (avoidance of thoughts and memories, etc.), which is one of the three PTSD diagnostic criteria. PTSD 'caseness' was therefore solely defined by criteria \#1 and \#3, which means that the number of PTSD cases was likely to have been overestimated, and some participants who met criteria \#1 and \#3 are likely to have been incorrectly labelled as PTSD cases. Whereas BPD diagnostic criteria are adequately described

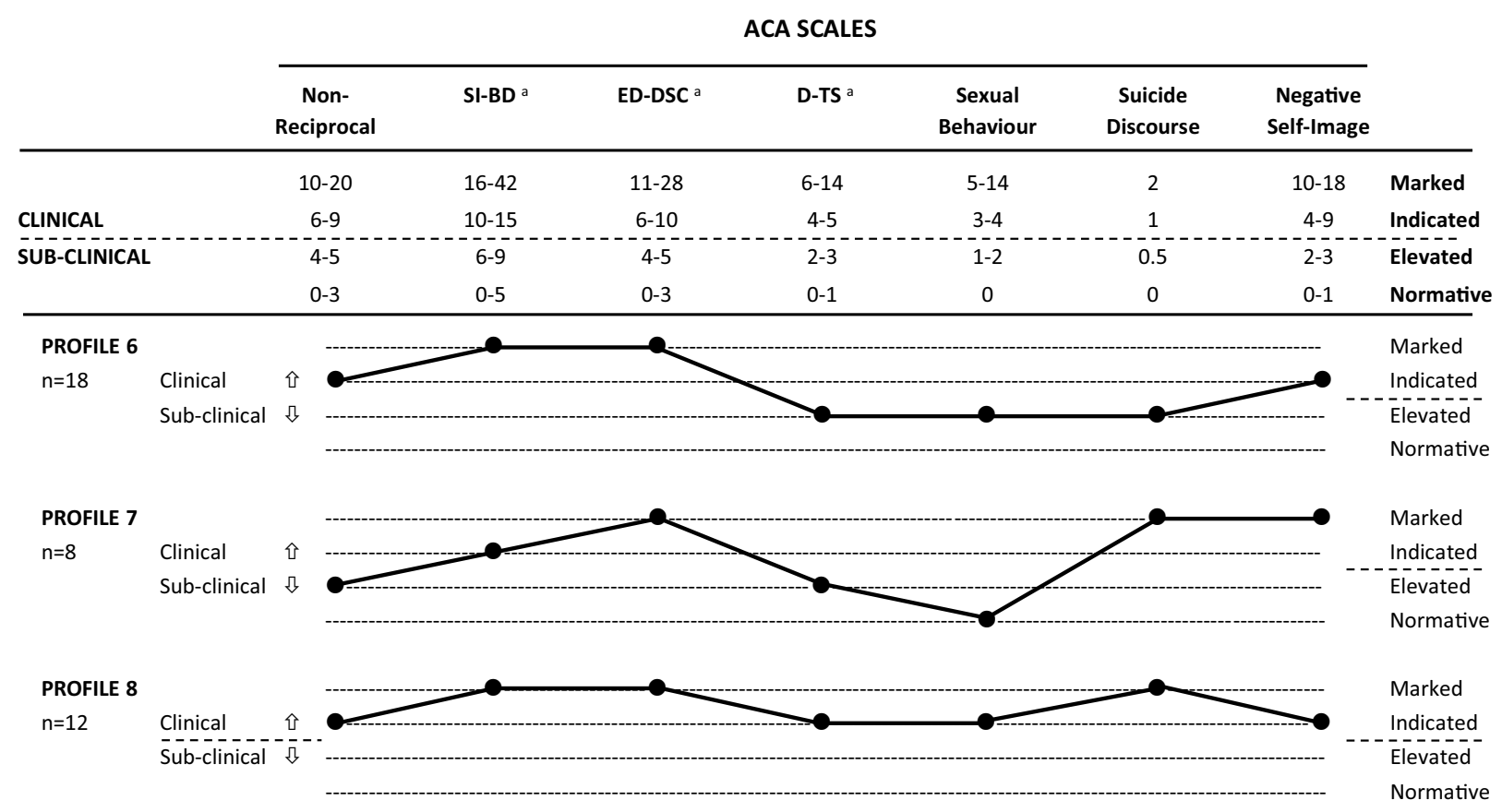

${ }^{\text {a }}$ SI-BD = Social instability / Behavioural dysregulation; ED-DSC = Emotional dysregulation / Distorted social cognition; D-TS $=$ Dissociation $/$ Trauma symptoms

Fig. 1 ACA symptom profiles \#6, \#7, and \#8 ( Source: Tarren-Sweeney, 2021) 


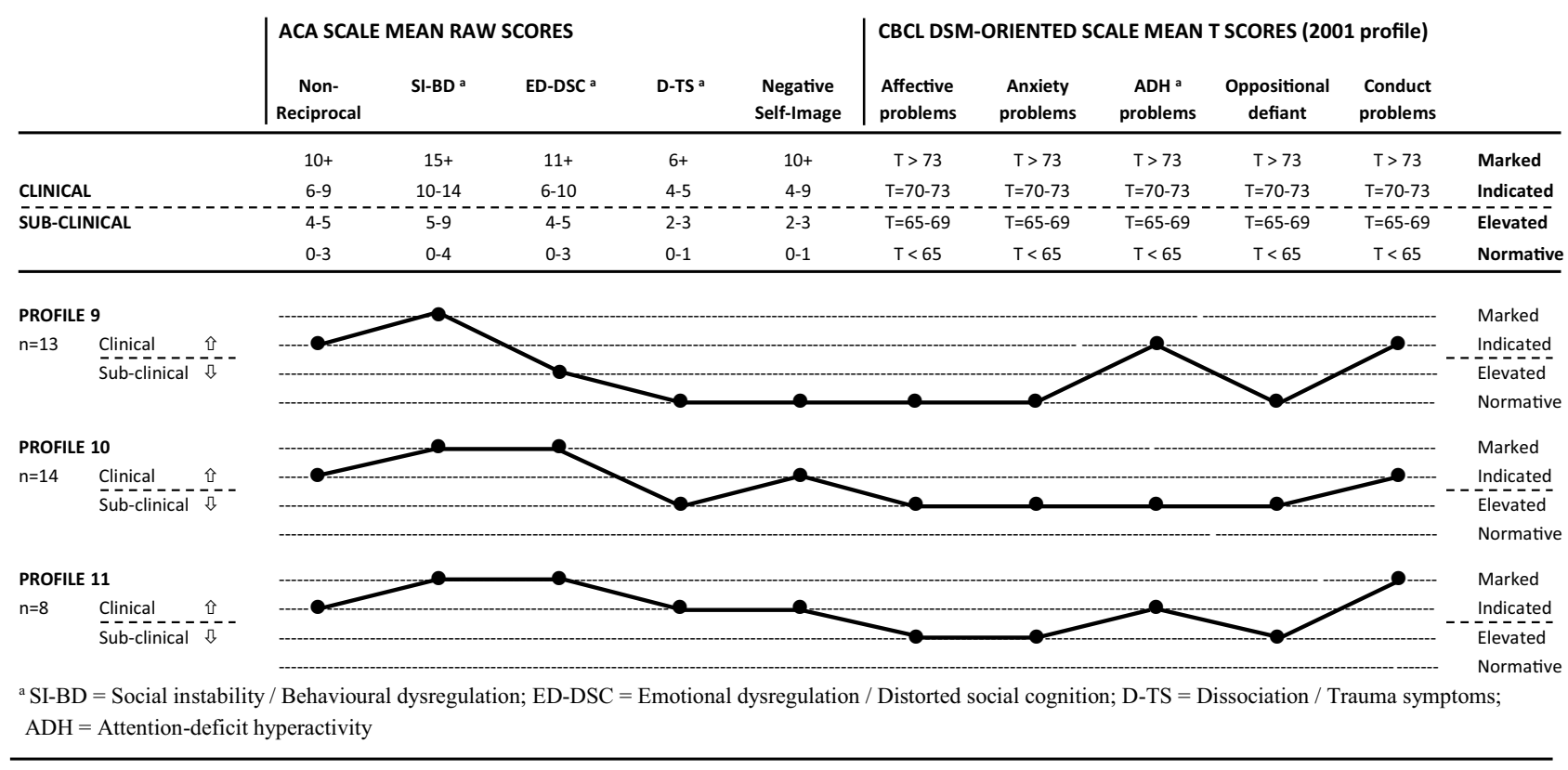

Fig. 2 CBCL-DSM/ACA symptom profiles \#9, \#10, and \#11 ( Source: Tarren-Sweeney, 2021)

in DSM-V, the C-PTSD diagnostic criteria listed on the ICD-11 website lack operational definitions, notably for the DSO criterion 'difficulties in sustaining relationships and in feeling close to others'. We therefore applied the description listed by Cloitre et al. (2013, pp. 2-3) namely "individuals may consistently avoid, deride or have little interest in relationships and social engagement more generally. The person may occasionally experience close or intense relationships but will have difficulty maintaining emotional engagement". The BPD diagnostic criteria are listed with the items selected to measure each criterion in Table 1, while the C-PTSD diagnostic criteria are listed with their selected items in Table 2.

The second step was to generate continuous scores for each criterion by adding the item scores. Each criterion thus had one continuous measure (i.e. a criterion scale), with the exceptions of BPD criterion \#9 and C-PTSD criterion \#4, which each required two criterion scales. Given that BPD criterion \#9 is met if 'transient, stress-related paranoid ideation or severe dissociative symptoms' occur, we created separate scales to measure paranoid ideation and severe dissociative symptoms. Similarly, C-PTSD criterion \#4 (Severe and pervasive problems in affect regulation) specifies two types of affect dysregulation ('under-active / dissociation' and 'over-active'), and thus separate scales were created for both types.

The third step was to select a cut-point for each criterion scale score that defined if the diagnostic criterion is met or not. Cut-points were selected conservatively, with a view to identifying probable rather than possible cases. Cut-point selection was guided by examining the number of items in the respective criterion scale, the scale score distributions, and clinical judgement about item quality (e.g. item specificity for the criterion). For BPD criterion \#9 and C-PTSD criterion \#4, the criterion was met if either or both of the criterion sub-types were met.

Finally, nominal BPD and C-PTSD cases were defined by applying the relevant diagnostic rules. DSM-V BPD diagnosis is defined as meeting five or more BPD diagnostic criteria, whereas C-PTSD diagnosis requires that all five of the measured criteria are met. In addition to defining C-PTSD cases, we also separately defined PTSD caseness (criteria $\# 1$ and \#3) and 'disturbances in self organisation' (DSO) caseness (criteria \#4, \#5 and \#6). Statistical analyses were performed with STATA - version 16 (StataCorp, 2019).

\section{Results}

BPD and C-PTSD criterion score means, diagnostic cutpoints, and numbers and rates of participants meeting criteria thresholds, are listed in Table 3. Rates of participants meeting criteria thresholds ranged from 2.2\% (BPD \#7 'chronic feelings of emptiness') to $30 \%$ (C-PTSD \#4 'affect dysregulation'). The proportions of participants who met zero through to nine BPD diagnostic criteria, and zero through to five C-PTSD criteria are listed in Table 4. For both disorders, more than half of the participants met zero diagnostic criteria. Nineteen nominal BPD cases (those who met five or more diagnostic criteria) and three nominal C-PTSD cases (those who met all five diagnostic criteria) were identified. 
Table 1 Items selected for BPD diagnostic criteria scales

\section{BPD diagnostic criteria}

(1) Frantic efforts to avoid real or imagined abandonment

(2) Pattern of unstable and intense interpersonal relationships characterized by alternating between extremes of idealization and devaluation

(3) Identity disturbance: markedly and persistently unstable self-image or sense of self

(4) Impulsivity in at least two areas that are potentially self-damaging

(5) Recurrent suicidal behaviour, gestures, or threats, or self-mutilating behaviour

(6) Affective instability due to a marked reactivity of mood (e.g., intense episodic dysphoria, irritability, or anxiety usually lasting a few hours and only rarely more than a few days)

\section{(7) Chronic feelings of emptiness}

(8) Inappropriate, intense anger or difficulty controlling anger (e.g., frequent displays of temper, constant anger, recurrent physical fights)

(9) Transient, stress-related paranoid ideation or severe dissociative symptoms

\section{Criterion scale items $^{\mathrm{a}}$}

Extreme reaction to losing a friend, or being excluded by other young people $^{\mathrm{b}}$

Fears you (or other adults) will reject him/her

Is convinced that friends will reject him/her

Possessive, can't share friends ${ }^{b}$

Changes friends quickly

Extreme reaction to losing a friend, or being excluded by other young people $^{b}$

Possessive, can't share friends ${ }^{b}$

Turns friends against each other

Seems like a completely different person (dramatic change in personality) (pilot item)

Thinks he/she is someone or something else (pilot item)

Constantly seeking excitement or 'thrills'

Impulsive (acts rashly, without thinking)

Risks physical safety, fearless

Attempts suicide

Causes injury to him/herself

Describes how he/she would kill him/herself

Hits head, head-banging

Intentionally harms him/herself with knife or sharp implement

Threatens to injure him/herself

Threatens to kill him/herself

Talks about killing self $(C B C L)$

Extreme reaction to minor event (or for no obvious reason)

Extreme reaction to losing a friend, or being excluded by other young people $^{\mathrm{b}}$

Intense reaction to criticism

Sudden or extreme mood changes

Says he/she feels 'empty' or without emotions

Shows intense and inappropriate anger

Uncontrollable rage

Temper tantrums or hot temper $(C B C L)$

A: Paranoid ideation:

Feels others are out to get him/her $(C B C L)$

Says friends are against him/her

$B$ : Severe dissociative symptoms

Appears dazed, 'spaced out' (like in a trance)

Can't tell if an experience is real or a dream

Feels like things, people or events aren't real

Has periods of amnesia (e.g. has no memory of what has happened in the last hour)

a ACA items except where indicated in brackets. 'CBCL' indicates Child Behavior Checklist item; 'Pilot item' refers to item included in initial development study that was not retained in final version of the ACA

${ }^{\mathrm{b}}$ Item included in more than one criterion scale

Table 5 reveals very little overlap between PTSD and DSO caseness. Among 22 participants who were PTSD and/ or DSO cases, there were only three C-PTSD cases. BPD caseness is tabulated against PTSD, DSO and C-PTSD caseness in Table 6 . This reveals moderately high overlap between DSO and BPD case assignment (62\% of DSO cases were also BPD cases), and somewhat lower overlap between PTSD and BPD case assignment (25\% of PTSD cases were also BPD cases). Two of the three C-PTSD cases were also BPD cases.
There are too few C-PTSD cases to report summary data. BPD cases and non-cases had the same mean age of 15.3 years. BPD prevalence among boys and girls was $6.4 \%(8 / 125)$ and $10.5 \%(11 / 105)$ respectively $\left(\mathrm{Chi}^{2}=1.3, \mathrm{p}=0.26\right)$. $\mathrm{BPD}$ cases had exceptionally high mean mental health difficulties, both in absolute terms and in comparison with the other clinical cases in the present study. Among cases selected for the ACA-CBCL cluster analysis $(N=141)$, mean ACA total clinical scores for BPD cases versus other cases were 68.8 and 26.3 respectively $(\mathrm{p}<0.0001)$, while mean CBCL total problems scores were 
Table 2 Items selected for C-PTSD diagnostic criteria scales

\section{C-PTSD diagnostic criteria}

\section{A.PTSD criteria}

(1) Re-experiencing traumatic event or events in the present in the form of vivid intrusive memories, flashbacks or nightmares. These are typically accompanied by strong or overwhelming emotions, particularly fear or horror, and strong physical sensations

(2) Avoidance of thoughts and memories of the event or events, or avoidance of activities, situations, or people reminiscent of the event or events

(3) Persistent perceptions of heightened current threat, for example as indicated by hypervigilance or an enhanced startle reaction to stimuli such as unexpected noises

\section{B.Disturbances in self organisation criteria}

(4) Severe and pervasive problems in affect regulation

(5) Persistent beliefs about oneself as diminished, defeated or worthless, accompanied by deep and pervasive feelings of shame, guilt or failure related to the traumatic event

(6) Persistent difficulties in sustaining relationships and in feeling close to others

\section{Criterion scale items}

Can't get scary thoughts or images out of his/her head

Distressed or troubled by traumatic memories

Nightmares about specific events or people

Nil items

Fears he/she might be molested (pilot item)

Is fearful of being harmed

Startles easily ('jumpy')

Wary or vigilant (over-alert to danger)

\author{
A. Over-reactive \\ Causes injury to him/herself \\ Extreme emotional reaction to minor event (or for no obvious reason) \\ Intentionally harms him/herself with knife/implement \\ Shows intense and inappropriate anger \\ Sudden or extreme mood changes \\ Temper tantrums or hot temper $(C B C L)$ \\ Uncontrollable rage \\ B. Under-reactive / dissociation \\ Appears dazed, 'spaced out' (like in a trance) \\ Can't tell if an experience is real or a dream \\ Does not show pain if physically hurt \\ Feels like things, people or events aren't real \\ Says he/she feels 'empty' or without emotions \\ Complains of not being likeable \\ Feels ashamed \\ Feels too guilty $(C B C L)$ \\ Feels worthless or inferior \\ Says he/she is 'bad' or 'no good' \\ Thinks other young people are better than him/her \\ Changes friends quickly \\ Distrusts friends \\ Does not show affection \\ Seems alone in the world (not connected to people or places \\ Won't communicate with other young people (pilot item)
}

a ACA items except where indicated in brackets. 'CBCL' indicates Child Behavior Checklist item; 'Pilot item' refers to item included in initial development study that was not retained in final version of the ACA

102.2 versus 54.0 respectively $(\mathrm{p}<0.0001)$. Based on caregivers' reports, twice as many BPD cases were prescribed psychotropic medication than were other clinical cases $(59 \%$ and $30 \%$ respectively, $p=0.02$ ).

Alignment of BPD and C-PTSD nominal diagnoses with the empirically derived ACA and DSM-CBCL/ACA symptom profiles are shown in Tables 7 and 8 respectively.

\section{Discussion}

As stated previously, the ACA and CBCL-DSM/ACA symptom profiles are differentiated more by symptom severity and complexity, than symptom specificity - suggesting that complex attachment- and trauma-related psychopathology is not amenable to traditional diagnostic classification. In other words, the profiles do not have sufficient symptom specificity to suggest a taxonomy of complex post-maltreatment developmental disorders. However, they offer an opportunity to test the validity of the BPD and C-PTSD constructs, against empirical, atheoretical symptom profiles. The ACA was designed to measure a broad range of maltreatmentrelated symptoms not adequately covered by general mental health measures such as the CBCL (Tarren-Sweeney, 2013a). The ACA symptom profiles were identified empirically through cluster analyses of the ACA scales, which in turn had been derived through factor analysis. The CBCLDSM/ACA symptom profiles are less empirically 'pure', in 
Table 3 BPD and C-PTSD criterion score means, diagnostic cut-points and assignment rates $(\mathrm{N}=230)$

\begin{tabular}{|c|c|c|c|c|c|}
\hline \multirow[t]{2}{*}{ Diagnostic criteria } & \multirow[t]{2}{*}{$\begin{array}{l}\text { N. criterion } \\
\text { scale items } \\
\left(\text { max score range }{ }^{a}\right)\end{array}$} & \multirow[t]{2}{*}{$\begin{array}{l}\text { Mean criterion } \\
\text { score }\end{array}$} & \multirow[t]{2}{*}{$\begin{array}{l}\text { Diagnostic } \\
\text { cut-point }\end{array}$} & \multicolumn{2}{|c|}{$\begin{array}{l}\text { Participants } \\
\text { meeting } \\
\text { criterion } \\
\text { threshold }\end{array}$} \\
\hline & & & & $\mathbf{N}$ & Rate \\
\hline \multicolumn{6}{|l|}{$B P D$ criteria } \\
\hline (1) Attempts to avoid real or imagined abandonment & $4(0-8)$ & 0.84 & $3+$ & 26 & $11.3 \%$ \\
\hline (2) Unstable and intense interpersonal relationships & $4(0-8)$ & 0.82 & $3+$ & 25 & $10.9 \%$ \\
\hline (3) Identity disturbance & $2(0-4)$ & 0.19 & $2+$ & 13 & $5.7 \%$ \\
\hline (4) Self-damaging impulsivity & $3(0-6)$ & 1.46 & $3+$ & 52 & $22.6 \%$ \\
\hline (5) Recurrent suicidal / self-mutilating & $8(0-16)$ & 0.52 & $2+$ & 23 & $10.0 \%$ \\
\hline (6) Affective instability due to marked mood reactivity & $4(0-8)$ & 1.48 & $4+$ & 38 & $16.5 \%$ \\
\hline (7) Chronic feelings of emptiness & $1(0-2)$ & 0.03 & $1+$ & 5 & $2.2 \%$ \\
\hline (8) Inappropriate, intense anger / difficulty controlling & $3(0-6)$ & 1.57 & $4+$ & 39 & $17.0 \%$ \\
\hline (9) Transient, stress-related paranoia or dissociation & & & $9 \mathrm{a} O R 9 \mathrm{~b}$ & 49 & $21.3 \%$ \\
\hline (9a) Paranoid ideation & $2(0-4)$ & 0.57 & $2+$ & 39 & $17.0 \%$ \\
\hline (9b) Dissociative symptoms & $4(0-8)$ & 0.62 & $3+$ & 18 & $7.8 \%$ \\
\hline \multicolumn{6}{|l|}{ C-PTSD criteria } \\
\hline \multicolumn{6}{|l|}{ A. PTSD } \\
\hline (1) Re-experiencing traumatic event(s) & $3(0-6)$ & 0.50 & $2+$ & 28 & $12.2 \%$ \\
\hline (2) Avoidance of traumatic event(s) & No scale & & & & \\
\hline (3) Persistent perceptions of heightened current threat & $4(0-8)$ & 0.55 & $2+$ & 37 & $16.1 \%$ \\
\hline \multicolumn{6}{|l|}{ B. Disturbances in self organisation } \\
\hline (4) Severe and pervasive problems in affect regulation & & & $4 \mathrm{a} O R 4 \mathrm{~b}$ & 69 & $30.0 \%$ \\
\hline (4a) Over-reactive affect & $7(0-14)$ & 2.43 & $4+$ & 61 & $21.7 \%$ \\
\hline (4b) Under-reactive affect / dissociation & $5(0-10)$ & 0.67 & $3+$ & 18 & $7.8 \%$ \\
\hline (5) Negative self-evaluation & $6(0-12)$ & 1.04 & $4+$ & 20 & $8.7 \%$ \\
\hline (6) Difficulties sustaining relationships & $5(0-10)$ & 1.46 & $4+$ & 25 & $8.9 \%$ \\
\hline
\end{tabular}

a'Max score range' refers to the maximum possible scale score range, given that items are scored 0,1 ,or 2

that they were constructed from scores on five ACA scales and five CBCL DSM-oriented scales, with the latter being derived deductively rather than inductively.

The present findings do not support the validity of the C-PTSD construct for describing complex and severe psychopathology among adolescents with a history of maltreatment. Despite the concern that our method may have over-estimated PTSD caseness by defining cases from only two of the three diagnostic criteria, just $1.3 \%$ of this highrisk adolescent sample were nominal C-PTSD cases. What
Table 4 Number of BPD and C-PTSD diagnostic criteria met $(\mathrm{N}=230)$

\begin{tabular}{lllllr}
\hline $\begin{array}{l}\text { N. BPD } \\
\text { criteria }\end{array}$ & $\mathrm{N}$ & $\%$ & $\begin{array}{l}\text { N. C-PTSD } \\
\text { criteria }\end{array}$ & $\mathrm{N}$ & $\%$ \\
\hline 0 & 129 & $56.1 \%$ & 0 & 123 & $53.5 \%$ \\
1 & 39 & $18.0 \%$ & 1 & 40 & $17.4 \%$ \\
2 & 23 & $10.0 \%$ & 2 & 36 & $15.7 \%$ \\
3 & 8 & $3.5 \%$ & 3 & 19 & $8.3 \%$ \\
4 & 12 & $5.2 \%$ & 4 & 9 & $3.9 \%$ \\
Diagnostic threshold & & Diagnostic threshold & & \\
5 & 10 & $4.4 \%$ & 5 & 3 & $1.3 \%$ \\
6 & 2 & $0.9 \%$ & & & \\
7 & 5 & $2.2 \%$ & & & \\
8 & 2 & $0.9 \%$ & & & \\
9 & 0 & $0.0 \%$ & & & \\
\hline
\end{tabular}


Table 5 Number of participants meeting PTSD and DSO diagnostic thresholds $(\mathrm{N}=230)$

\begin{tabular}{lllll}
\hline & \multicolumn{4}{l}{ PTSD threshold } \\
\hline DSO threshold & & Not met & Met & Total \\
& Not met & 208 & 9 & 217 \\
& Met & 10 & 3 & 13 \\
& Total & 218 & 12 & 230 \\
\hline
\end{tabular}

might explain this? One possibility is that our method underestimated the number of participants meeting one or more diagnostic criteria, due to cut-points being set too high, and/ or a lack of adequately matched items. This is critical given that C-PTSD diagnosis requires all of the diagnostic criteria to be met. However, the proportions of participants meeting the various diagnostic criteria ranged from 8 to $30 \%$ (see Table 3), suggesting there was no diagnostic 'trip point' causing underdetection of C-PTSD. For the PTSD criteria (\#1 and \#3), the relatively low $2+$ cut-points yielded fairly high criterion assignment rates of $12 \%$ and $16 \%$ respectively, with $23 \%$ of the sample $(N=53)$ meeting one or both PTSD criteria. Surprisingly however, less than a quarter of those $(23 \%, N=12)$ met both PTSD criteria. We also believe that the selected ACA and CBCL items matched the C-PTSD criteria well. Instead, the very low nominal C-PTSD prevalence appears to be accounted for by low concordance across the five criteria, and more particularly, between PTSD and DSO caseness.

The present findings suggest that the core features of BPD (emotional and behavioural dysregulation, including impulsivity and intense anger; intense and unstable interpersonal relationships underpinned by fears of rejection and abandonment; disturbance of 'self' with negative self-image; dissociation and paranoia; and suicidality) are components of severe and complex developmental symptomatology experienced by adolescents who experienced early social adversity, including maltreatment. Of the 19 nominal BPD cases, 18 belonged to the three most severe and complex ACA clusters (ACA profiles \#6, \#7, \#8). The most striking finding is that all 12 participants whose symptoms fitted ACA symptom profile \#8 (the most severe and complex profile) were BPD

Table 6 Overlap between BPD and C-PTSD case assignment

\begin{tabular}{|c|c|c|c|}
\hline & & $\begin{array}{l}\text { BPD case } \\
\text { assignment }\end{array}$ & \\
\hline \multirow{5}{*}{$\begin{array}{l}\text { C-PTSD } \\
\text { case } \\
\text { assignment }\end{array}$} & & Non-case & Case \\
\hline & $\begin{array}{l}\text { Meets neither } \\
d x \text { threshold }(N=208)\end{array}$ & 198 & 10 \\
\hline & $\begin{array}{l}\text { Meets PTSD } \\
d x \text { threshold }(N=12)\end{array}$ & 9 & 3 \\
\hline & $\begin{array}{l}\text { Meets DSO } \\
\text { dx threshold }(N=13)\end{array}$ & 5 & 8 \\
\hline & $C$-PTSD cases $(N=3)$ & 1 & 2 \\
\hline
\end{tabular}

Table 7 Alignment of BPD and C-PTSD diagnoses with ACA symptom profiles

\begin{tabular}{llll}
\hline Profile & $\begin{array}{l}\text { Total ACA cases } \\
(N=113)\end{array}$ & $\begin{array}{l}\text { BPD cases } \\
(N=19)\end{array}$ & $\begin{array}{l}\text { C-PTSD } \\
\text { cases } \\
(N=3)\end{array}$ \\
\hline$\# 1$ & 17 & 0 & 0 \\
$\# 2$ & 21 & 0 & 0 \\
$\# 3$ & 13 & 0 & 0 \\
$\# 4$ & 15 & 1 & 0 \\
$\# 5$ & 9 & 0 & 0 \\
$\# 6$ & 18 & 4 & 1 \\
$\# 7$ & 8 & 2 & 0 \\
$\# 8$ & 12 & 12 & 2 \\
\hline
\end{tabular}

cases, inferring that ACA profile \#8 corresponds to severe or pronounced BPD. There was similar alignment of BPD caseness with the CBCL-DSM/ACA symptom profiles. Of the 19 BPD cases, 15 were assigned to CBCL-DSM/ACA symptom profiles \#10 and \#11 (the two most severe and complex profiles), and all eight participants that had the most severe profile (\#11) were BPD cases, inferring that ACA-CBCL profile \#11 corresponds to severe or pronounced BPD. The ACA symptom profiles \#6 and \#8 were characterised by cooccurring non-reciprocal social behaviour and intense and unstable interpersonal relationships underpinned by fears of rejection and abandonment (as measured by the SI-BD and ED-DSC scales). While these two sets of behaviours appear contradictory, they are consistent with a characteristic relational pattern observed among individuals with BPD, namely rapid alternation between craving contact and affection, and rejecting contact - and corresponding shifts between extremes of idealization and devaluation (Newman \& Stevenson, 2005).

Table 8 Alignment of BPD and C-PTSD diagnoses with CBCLDSM/ACA symptom profiles

\begin{tabular}{llll}
\hline Profile & $\begin{array}{l}\text { Total CBCL-DSM } \\
\text { / ACA } \\
\text { cases }(N=141)\end{array}$ & $\begin{array}{l}\text { BPD cases } \\
(N=19)\end{array}$ & $\begin{array}{l}\text { C-PTSD } \\
\text { cases } \\
(N=3)\end{array}$ \\
\hline$\# 1$ & 13 & 0 & 0 \\
$\# 2$ & 23 & 0 & 0 \\
$\# 3$ & 12 & 0 & 0 \\
$\# 4$ & 15 & 0 & 0 \\
$\# 5$ & 9 & 0 & 0 \\
$\# 6$ & 8 & 1 & 0 \\
$\# 7$ & 8 & 1 & 0 \\
$\# 8$ & 18 & 1 & 0 \\
$\# 9$ & 13 & 1 & 0 \\
$\# 10$ & 14 & 7 & 2 \\
$\# 11$ & 8 & 8 & 1 \\
\hline
\end{tabular}




\section{Limitations and Implications for Future Research}

The present study findings are based solely on caregiverreported mental health checklist scores. Further research on this topic should ideally include adolescent clinical interviews and self-report measures, as well as caregiverreport measures. However, it is important to retain caregiver measures in any future study, given evidence that young people in OOHC systematically under-report their mental health difficulties in population studies (TarrenSweeney, 2019). A second possible study limitation is that BPD and C-PTSD cases were defined from items on broad-range mental health checklists, rather than instruments designed explicitly to measure BPD and C-PTSD diagnostic criteria. However, with the exception of C-PTSD criterion \#2 (avoidance of thoughts and memories), we believe the items selected from the CBCL and ACA were, in the main, well matched to the various diagnostic criteria.

The present study focussed exclusively on the mental health outcomes of adolescents in foster care, without referencing other developmental outcomes, notably cognitive abilities and language. Neurocognitive deficits are likely to increase chronically maltreated children's vulnerability for complex and severe mental health difficulties. Future investigations of complex symptomatology with maltreated children and adolescents would ideally include formal cognitive assessment, as well as neuropsychological, adaptive behaviour and language measures. Furthermore, longitudinal studies are needed to account for and disentangle complex transactional developmental processes affecting this population, including differentiating the effects of pre-care maltreatment from within-care adversity (e.g. placement instability, and relational impermanence), as well as the overlaying influence of emerging mental illness in adolescence.

\section{Practice Implications}

Accurate assessment and formulation of complex symptomology among this vulnerable population is critical for treatment planning. The present study findings highlight the limitations of the ICD and DSM classification systems for young people with complex maltreatment-related symptomatology. While the terms 'complex trauma' and 'developmental trauma' are increasingly used to describe the clinical-developmental effects of early and prolonged maltreatment, the present study findings suggest a need for caution when diagnosing 'trauma' disorders among maltreated young people with complex difficulties. In particular, a thorough assessment of attachment-related relational difficulties and styles is necessary before diagnosing C-PTSD.

\section{Conclusion}

Our investigation of alignment between empirically-derived symptom profiles and nominal diagnosis of DSM-V BPD and DSM-11 C-PTSD provides some support for the validity of the BPD construct among adolescents exposed to early maltreatment - but no support for the C-PTSD construct. While the complex and severe presentations identified in the present study included core symptoms and clinical signs of BPD, they were also characterised by clinical-level inattention/over-activity and conduct problems. The complex symptom profiles thus point to symptomatology that is broader and more complex than that encapsulated by the BPD construct. The present results therefore suggest that, while BPD as it is presently conceptualised describes a large portion of symptoms present in complex and severe adolescent presentations, it fails to account for co-occurring clinical-level conduct problems and inattention/over-activity (see Fig. 2). Given the high rate of co-morbid diagnosis of BPD with other developmental disorders (including ADHD) among adults, the present findings suggest that BPD may not be accurately conceptualised for adults who experienced childhood maltreatment. Otherwise, might the present findings point to the existence of an adolescent complex disorder that incorporates core features of Conduct Disorder, ADHD and BPD? While this is a tempting possibility, not least because it would provide clinicians a discrete diagnostic formulation that is unencumbered by co-morbidity, it is compromised by very high variability in combinations of multiple symptom types that does not conform to traditional definitions of a 'diagnosable' mental disorder. Furthermore, there may be greater utility in conceptualizing complex presentations among this population using a 'symptom profile' or 'complex developmental formulation' approach, in place of a traditional diagnostic formulation - that emphasises a trans-diagnostic focus on transactional developmental mechanisms of early caregiving, attachment, traumatic abuse, social learning conditions and biological vulnerability for emerging mental illness.

Acknowledgements This study was funded and supported by the NSW Department of Communities and Justice. Professor Ken Nunn, of Westmead Children's Hospital and the Elver Trauma Service (Sydney, Australia), provided invaluable advice for our preparation of this article.

Funding Open Access funding enabled and organized by CAUL and its Member Institutions.

\section{Declarations}

Informed Consent Adolescent participants and their caregivers separately provided informed consent for caregivers to complete the survey questionnaire and for the research team to access their child welfare records. 


\section{Ethical Approval}

The study was approved by the Human Ethics Committees of the University of Newcastle, Australia (H-2008-0256) and the University of Canterbury, New Zealand (HEC-2008/93). All procedures performed were in accordance with the ethical standards of the institutional research committees and with the 1964 Helsinki declaration and its later amendments or comparable ethical standards.

Conflict of Interest The authors have no relevant financial or non-financial interests to disclose.

Open Access This article is licensed under a Creative Commons Attribution 4.0 International License, which permits use, sharing, adaptation, distribution and reproduction in any medium or format, as long as you give appropriate credit to the original author(s) and the source, provide a link to the Creative Commons licence, and indicate if changes were made. The images or other third party material in this article are included in the article's Creative Commons licence, unless indicated otherwise in a credit line to the material. If material is not included in the article's Creative Commons licence and your intended use is not permitted by statutory regulation or exceeds the permitted use, you will need to obtain permission directly from the copyright holder. To view a copy of this licence, visit http://creativecommons.org/licenses/by/4.0/.

\section{References}

Achenbach, T., \& Rescorla, L. (2001). Manual for ASEBA school-age forms and profiles. University of Vermont, Research Center for Children, Youth, \& Families.

Ad-Dab'bagh, Y., \& Greenfield, B. (2001). Multiple complex developmental disorder: The "multiple and complex" evolution of the "Childhood Borderline Syndrome" construct. Journal of the American Academy of Child \& Adolescent Psychiatry, 40(8), 954-964.

American Psychiatric Association. (1994). Diagnostic and statistical manual of mental disorders. (4th ed.). Washington D.C.: American Psychiatric Association.

American Psychiatric Association. (2013). Diagnostic and statistical manual of mental disorders (5th ed.): https://doi.org/10.1176/appi. books. 9780890425596

Belsky, D., Caspi, A., Arseneault, L., Bleidorn, W., Fonagy, P., Goodman, M., \& Moffitt, T. (2012). Etiological features of borderline personality related characteristics in a birth cohort of 12 year old children. Development and Psychopathology, 24(1), 251-265. https://doi.org/ 10.1017/S0954579411000812

Boris, N. W., \& Zeanah C. (1999). Disturbances and disorders of attachment in infancy: An overview. Infant Mental Health Journal, 20(1), 1-9.

Brand, B. L., \& Lanius, R. A. (2014). Chronic complex dissociative disorders and borderline personality disorder: disorders of emotion dysregulation? Borderline Personality Disorder and Emotion Dysregulation, 1(13). https://doi.org/10.1186/2051-6673-1-13

Brewin, C., Cloitre, M., Hyland, P., Shevlin, M., Maercker, A., Bryant, R., \& Reed, G. (2017). A review of current evidence regarding the ICD-11 proposals for diagnosing PTSD and complex PTSD. Clinical Psychology Review, 58, 1-15.

Chanen, A., Jackson, H., McGorry, P., Allot, K., Clarkson, V., \& Hok, P. (2004). Two-year stability of personality disorder in older adolescent outpatients. Journal of Personality Disorders, 18(6), 526-541.

Cloitre, M, Garvert, D, Brewin, C, Bryant, R, \& Maercker, A. (2013). Evidence for proposed ICD-11 PTSD and complex PTSD: A latent profile analysis European Journal of Psychotraumatology, 4. https://doi.org/10.3402/ejpt.v4i0.20706
Cloitre, M., Garvert, D. W., Weiss, B., Carlson, E. B., \& Bryant, R. A. (2014). Distinguishing PTSD, complex PTSD, and borderline personality disorder: A latent class analysis. European journal of psychotraumatology, 5(1), 25097. https://doi.org/10.3402/ejpt.v5.25097

Cloitre, M., Stolbach, B., Herman, J., Van Der Kolk, B., Pynoos, R., Wang, J., \& Petkova, E. (2009). A developmental approach to complex PTSD: Childhood and adult cumulative trauma as predictors of symptom complexity (Conference Paper). Journal of Traumatic Stress, 22(5), 399-408. https://doi.org/10.1002/jts.20444

Cohen, D., Volkmar, F., \& Paul, R. (1986). Issues in the classification of pervasive developmental disorders: History and current status of nosology. Journal of the American Academy of Child \& Adolescent Psychiatry, 25(2), 158-161.

Cook, A., Spinazzola, J., Ford, J., Lanktree, C., Blaustein, M., Cloitre, M., \& van der Kolk, B. (2005). Complex trauma in children and adolescents. Psychiatric Annals, 35(5), 390-398.

Creamer, M., Burgess, P., \& McFarlane, A. (2001). Posttraumatic stress disorder: findings from the Australian national survey of mental health and well-being. Psychological Medicine, 31(7), 1237-1247.

D’Andrea, W., Ford, J., Stolbach, B., Spinazzola, J., \& van der Kolk, B. (2012). Understanding interpersonal trauma in children: Why we need a developmentally appropriate trauma diagnosis. American Journal of Orthopsychiatry, 82(2), 187-200. https://doi.org/10. 1111/j.1939-0025.2012.01154.x

DeJong, M. (2010). Some reflections on the use of psychiatric diagnosis in the looked after or 'in care' child population. Clinical Child Psychology and Psychiatry, 15(4), 589-599. https://doi.org/10. 1177/1359104510377705

Fonagy, P., \& Bateman, A. (2006). Mechanisms of change in mentalization-based treatment of BPD. Journal of Clinical Psychology, 62(4), 411-430.

Ford, J. (2020). New findings questioning the construct validity of complex posttraumatic stress disorder (cPTSD): Let's take a closer look. European Journal of Psychotraumatology, 11, 1-7. https:// doi.org/10.1080/20008198.2019.1708145

Frías, A., Baltasar, I., \& Birmaher, B. (2016). Comorbidity between bipolar disorder and borderline personality disorder: Prevalence, explanatory theories, and clinical impact. Journal of Affective Disorders, 202, 210-219. https://doi.org/10.1016/j.jad.2016.05.048

Haselgruber, A., Sölva, K., \& Lueger-Schuster, B. (2020). Validation of ICD-11 PTSD and complex PTSD in foster children using the International Trauma Questionnaire. Acta Psychiatrica Scandinavica, 141, 60-73.

Helgeland, M. I., \& Torgersen, S. (2004). Developmental antecedents of borderline personality disorder. Comprehensive Psychiatry, 45(2), 138-147. https://doi.org/10.1016/j.comppsych.2003.09.001

Henry, D. B., Tolan, P. H., \& Gorman-Smith, D. (2005). Cluster analysis in family psychology research. Journal of Family Psychology, 19(1), 121-132. https://doi.org/10.1037/0893-3200.19.1.121

Herman, J. L. (1992). Complex PTSD: A syndrome in survivors of prolonged and repeated trauma. Journal of Traumatic Stress, 5(3), 377-391.

Infurna, M. R., Brunner, R., Holz, B., Parzer, P., Giannone, F., Reichl, C., \& Kaess, M. (2016). The specific role of childhood abuse, parental bonding, and family functioning in female adolescents with borderline personality disorder. Journal of Personality Disorders, 30(2), 177-192. https://doi.org/10.1521/pedi_2015_29_186

Kocovska, E., Puckering, C., Follan, M., Smillie, M., Gorski, C., Barnes, J., \& Minnis, H. (2012). Neurodevelopmental problems in maltreated children referred with indiscriminate friendliness. Research in Developmental Disabilities, 33(5), 1560-1565.

Levy, K., Becker, D., Grilo, C., Mattanah, J., Garnet, K., Quinlan, D., \& McGlashan, T. (1999). Concurrent and predictive validity of the 
personality disorder diagnosis in adolescent inpatients. American Journal of Psychiatry, 156(10), 1522-1528.

Lewinsohn, P., Rohde, P., Seeley, J., \& Klein, D. (1997). Axis II psychopathology as a function of Axis I disorders in childhood and adolescence. Journal of the American Academy of Child \& Adolescent Psychiatry, 36(12), 1752-1759.

McNab, C, McCutcheon, L, \& Chanen, A. (2021). Borderline Personality Disorder, stigma and young people: Research Bulletin. Melbourne, Australia: Orygen: National Centre of Excellence in Youth Mental Health.

Minnis, H. (2013). Maltreatment-associated psychiatric problems: An example of environmentally triggered ESSENCE? The Scientific World Journal. https://doi.org/10.1155/2013/148468

Newman, L., \& Stevenson, C. (2005). Parenting and borderline personality disorder: ghosts in the nursery. Clinical Child Psychology and Psychiatry, 10(3), 385-394. https://doi.org/10.1177/1359104505053756.

Pagura, J, Stein, M, Bolton, J, Cox, B, Grant, B, \& Sareen, J. (2010). Comorbidity of borderline personality disorder and posttraumatic stress disorder in the U.S. population. Journal of Psychiatric Research, 44(16), 1190-1198. https://doi.org/10.1016/j.jpsychires.2010.04.016

Perkonigg, A., Höfler, M., Cloitre, M., Wittchen, H., Trautmann, S., \& Maercker, A. (2016). Evidence for two different ICD-11 posttraumatic stress disorders in a community sample of adolescents and young adults. European Archives of Psychiatry and Clinical Neuroscience, 266, 317-328. https://doi.org/10.1007/ s00406-015-0639-4

Sachser, C., Keller, F., \& Goldbeck, L. (2017). Complex PTSD as proposed for ICD-11: Validation of a new disorder in children and adolescents and their response to Trauma-Focused Cognitive Behavioral Therapy. Journal of Child Psychology and Psychiatry, 58(2), 160-168. https://doi.org/10.1111/jcpp.12640

Sansone, R., \& Sansone, L. (2013). Responses of mental health clinicians to patients with borderline personality disorder. Innovations in Clinical Neuroscience, 10(5-6), 39-43.

Schmid, M., Petermann, F., \& Fegert, J. M. (2013). Developmental trauma disorder: Pros and cons of including formal criteria in the psychiatric diagnostic systems. BMC Psychiatry, 13. https://doi. org/10.1186/1471-244X-13-3

Spinazzola, J., Blaustein, M., \& van der Kolk, B. (2005). Posttraumatic stress disorder treatment outcome research: The study of unrepresentative samples? Journal of Traumatic Stress, 18(5), 425-436.

StataCorp. (2019). Stata statistical software: Release 16 (Version 16.0) [Statistical software]. College Station, TX: StataCorp LP.

Steele, R., \& Aylward, B. (2007). The use of cluster analytic techniques in developmental and behavioral research. Journal of Developmental and Behavioral Pediatrics, 28(4), 327-329. https://doi. org/10.1097/dbp.0b013e3181132008

Tarren-Sweeney, M. (2007). The Assessment Checklist for Children ACC: A behavioral rating scale for children in foster, kinship and residential care. Children \& Youth Services Review, 29, 672-691. https://doi.org/10.1016/j.childyouth.2007.01.008

Tarren-Sweeney, M. (2013a). The Assessment Checklist for Adolescents - ACA: A scale for measuring the mental health of young people in foster, kinship, residential and adoptive care. Children and Youth Services Review, 35, 384-393. https://doi.org/10. 1016/j.childyouth.2012.12.005

Tarren-Sweeney, M. (2013b). An investigation of complex attachmentand trauma-related symptomatology among children in foster and kinship care. Child Psychiatry \& Human Development, 44, 727 741. https://doi.org/10.1007/s10578-013-0366-x

Tarren-Sweeney, M. (2016). The developmental case for adopting children from care. Clinical Child Psychology \& Psychiatry, 21(4), 497-505. https://doi.org/10.1177/1359104516670277

Tarren-Sweeney, M. (2018). The mental health of adolescents residing in court-ordered foster care: Findings from a population survey. Child Psychiatry and Human Development, 49, 443-451. https:// doi.org/10.1007/s10578-017-0763-7

Tarren-Sweeney M. (2019). Do adolescents in care systematically underreport their mental health difficulties in population studies? A narrative review Developmental Child Welfare. Online First, 1-22 https://doi.org/10.1177/2516103219829483

Tarren-Sweeney, M. (2021). Mental health symptom profiles of adolescents placed into foster care. Journal of Child \& Adolescent Trauma. https://doi.org/10.1007/s40653-021-00417-2

Towbin, K., Dykens, E., Pearson, G., \& Cohen, D. (1993). Conceptualizing "borderline syndrome of childhood" and "childhood schizophrenia" as a developmental disorder. Journal of the American Academy of Child and Adolescent Psychiatry, 32(4), 775-782. https://doi.org/10.1097/00004583-199307000-00011

Trull, T., Stepp, S., \& Durrett, C. (2003). Research on borderline personality disorder: An update. Current Opinion in Psychiatry, 16(10), 77-82.

van der Kolk, B. (2005). Developmental Trauma Disorder: Toward a rational diagnosis for children with complex trauma histories. Psychiatric Annals, 35(5), 401-408.

van Der Kolk, B. (2016). Commentary: The devastating effects of ignoring child maltreatment in psychiatry - A commentary on Teicher and Samson. Journal of Child Psychology and Psychiatry and Allied Disciplines, 57(3), 267-270.

van der Kolk, B., Roth, S., Pelcovitz, D., Sunday, S., \& Spinazzola, J. (2005). Disorders of extreme stress: The empirical foundation of a complex adaptation to trauma. Journal of Traumatic Stress, 18, 389-399. https://doi.org/10.1002/jts.20047

Venta, A., Kenkel-Mikelonis, R., \& Sharp, C. (2012). A preliminary study of the relation between trauma symptoms and emerging BPD in adolescent inpatients. Bulletin of the Menninger Clinic, 76(2), 130-146. https://doi.org/10.1521/bumc.2012.76.2.130

Weiner, L., Perroud, N., \& Weibel, S. (2019). Attention Deficit Hyperactivity Disorder and Borderline Personality Disorder in adults: A review of their links and risks. Neuropsychiatric Disease and Treatment, 15, 3115-3129.

World Health Organization. (2020). International statistical classification of diseases and related health problems (11th ed.): https:// icd.who.int/

Publisher's Note Springer Nature remains neutral with regard to jurisdictional claims in published maps and institutional affiliations. 